\title{
Predictors and Outcomes of Hospitalization for Influenza: Real-World Evidence from the United States Medicare Population
}

\author{
Susan C. Bolge $\cdot$ Furaha Kariburyo $\cdot$ Huseyin Yuce $\cdot$ Roman Fleischhackl
}

Received: June 5, 2020 / Accepted: October 6, 2020 / Published online: October 27, 2020

(C) The Author(s) 2020

\begin{abstract}
Introduction: The purpose of this study was to identify predictors of initial hospitalization and describe the outcomes of high-risk patients hospitalized with influenza.

Methods: Data were taken from the 5\% national US Medicare database from 2012 to 2015. Patients (aged at least 13 years) were required to have at least one diagnosis for influenza and have continuous health plan enrollment for 6 months before (baseline) and 3 months (follow-up) after the date of influenza diagnosis. Patients who died during follow-up were included. Patients were categorized as initially hospitalized if hospitalized within $0-1$ day of diagnosis. High-risk initially hospitalized
\end{abstract}

Electronic supplementary material The online version of this article (https://doi.org/10.1007/s40121020-00354-X) contains supplementary material, which is available to authorized users.

S. C. Bolge

Janssen Global Services, LLC, Raritan, NJ, USA

F. Kariburyo

SIMR, LLC, Ann Arbor, MI, USA

F. Kariburyo $(\bowtie) \cdot$ H. Yuce

New York City College of Technology, City

University of New York, New York, NY, USA

e-mail: fkariburyo@statinmed.com

R. Fleischhackl

Janssen-Cilag, Vienna, Austria patients were defined as patients aged at least 65 years or those that had a diagnostic code for chronic lung disease, cardiovascular or cerebrovascular disease, or weakened immune system during baseline period. Logistic regression models were developed to determine predictors of initial hospitalization.

Results: The study population included 8127 high-risk patients who were initially hospitalized and 16,784 who were not hospitalized. Among high-risk patients, $89.3 \%$ were diagnosed in the emergency room, whereas $7.5 \%$ and $3.2 \%$ were diagnosed in a physician's office or other Medicare settings, respectively. Chronic obstructive pulmonary disorder, congestive heart failure, chronic kidney disease, older age, being male, other comorbidities, number of comorbidities, and baseline healthcare resource use were the predictors of hospitalization. Median length of stay for the hospitalization was 5.0 days, and the 30-day readmission rate was $14 \%$. All-cause mortality rate was $5.1 \%$ during the inpatient stay and $9.2 \%$ within 30 days of diagnosis. Hospitalized patients with influenza incurred an increase of $\$ 16,568$ per patient in total all-cause healthcare costs from pre-influenza to post-influenza diagnosis.

Conclusion: The study characterized the burden of hospitalization for influenza and found that hospitalized high-risk patients experience greater comorbidity burden, higher likelihood of multiple inpatient admissions, and costly 
medical interventions compared to patients who were not hospitalized.

Keywords: Emergency hospitalization; Highrisk influenza; Window of treatment

\section{Key Summary Points}

\section{Why carry out this study?}

The burden of influenza remains significant for individuals at high risk of influenza complications, including those aged 65 years or older with underlying conditions.

It is of utmost importance for physicians to identify appropriate patients for treatment at the time of diagnosis.

The purpose of this study was to identify predictors of initial hospitalization and describe the current outcomes of high-risk patients hospitalized with influenza.

\section{What was learned from the study?}

Emergency departments are especially likely to be the first point of care for highrisk patients with influenza who require hospitalization.

Predictors that can help identify these patients are older age, greater comorbidity burden, and diagnosis of chronic obstructive pulmonary disease (COPD), congestive heart failure (CHF), or chronic kidney disease (CKD).

Hospitalized patients with influenza incurred an increase of $\$ 16,568$ per patient in total all-cause healthcare costs mostly due to increased all-cause hospitalization costs (from $\$ 4305$ to $\$ 16,651$ ) from pre-influenza to postinfluenza diagnosis.

\section{DIGITAL FEATURES}

This article is published with digital features, including a summary slide, to facilitate understanding of the article. To view digital features for this article go to https://doi.org/10.6084/ m9.figshare.13050497.

\section{INTRODUCTION}

Influenza is an acute respiratory infection caused by the influenza virus A and B and is highly contagious [1]. According to the World Health Organization, approximately 3-5 million cases of severe illness due to the influenza virus are reported annually [2]. Since 2010, annual influenza figures in the USA have ranged from 9.2 to 35.6 million total illnesses, 4.3-16.7 million medical visits, 140,000-710,000 hospitalizations, and 12,000-56,000 deaths [3]. In 2015 , the total economic burden of influenza was estimated at $\$ 11.2$ billion, out of which $\$ 3.2$ billion contributed to direct medical costs and $\$ 8.0$ billion was associated with indirect medical costs [4]. Adults aged 65 years or older contribute to the largest share of total direct costs resulting from hospitalizations. Karve et al. reported that the cost of complicated influenza was twice as high as uncomplicated influenza [5].

In most influenza cases, the infection is a self-limiting disease that resolves on its own without the risk of developing serious complications [6-8]. However, the burden of influenza remains significant for individuals at high risk of influenza complications, including those aged 65 years or older with underlying conditions such as respiratory disease, neurological disease, heart disease, hematological disorders, kidney/liver disorders, metabolic disorders, and weakened immune system [9-11].

Optimal influenza vaccinations are a public health priority because they can decrease 
influenza-related morbidity and mortality and also reduce economic burden by minimizing the number of missed workdays due to illness $[12,13]$. Although vaccination is the only recommended prevention strategy for populations at greater risk such as individuals aged 65 years or older in the USA, the vaccination uptake and coverage rates have historically fallen below the target of $70 \%$. In the USA influenza seasons from 2011 to 2012 through 2015-2016, yearly vaccination proportions remained around $65 \%$ among adults aged 65 years or older [14]. Overall influenza vaccine effectiveness for the general population has ranged widely, from $19 \%$ to $60 \%$ since the $2010-11$ season $[15,16]$.

As most influenza viruses have acquired resistance to M2 ion channel inhibitors, hospitalized patients and outpatients at high risk for serious complications from influenza are generally managed with antiviral medication-primarily neuraminidase inhibitors (NAIs) [17]. While resistant strains are a persistent challenge, NAIs remain the standard of care (during the time the study was conducted) and have been associated with improved clinical outcomes [18]. Although NAIs are most commonly used, the current recommended window for treatment initiation is less than $48 \mathrm{~h}$ from the onset of symptoms [19-21]. In addition, limited evidence is available concerning NAI treatment as related to significant improvement in patient outcomes, particularly for those in the hospital setting in patients with uncomplicated acute influenza. To date, there is no evidence of significant improvement in US patients who have started the treatment more than $48 \mathrm{~h}$ after symptoms began [22-24].

Because treatment may be more effective if initiated sooner, it is of utmost importance for physicians to identify appropriate patients for treatment at the time of diagnosis [25]. Influenza diagnostics are evolving, and influenza therapeutics currently in development are designed to address high-risk, complicated, and hospitalized patients [26]. Therefore, a key determinant will be to identify patients who need to be tested and treated early as well as those at risk of hospitalization in order to prevent poor outcomes and complications such as hospitalization. Therefore, the purpose of this study was to identify predictors of initial hospitalization and describe the current outcomes of high-risk patients hospitalized with influenza.

\section{METHODS}

\section{Data Source}

This real-world observational study was conducted using the Medicare 5\% national sample administrative database from January 2011 through December 2015. All patient identifiers in the database have been fully encrypted, and the database is fully compliant with the Health Insurance Portability and Accountability Act of 1996. The Medicare claims data files used for this study included inpatient and outpatient, Medicare carrier, Part D drug events, skilled nursing facility (SNF), home health agency, hospice, durable medical equipment, and Medicare denominator file (which contains demographic and enrollment information of Medicare beneficiaries).

\section{Patient Selection}

Patients with a medical claim for influenza diagnosis (International Classification of Disease, 9th Revision, Clinical Modification [ICD9-CM] codes 487, 488; ICD-10-CM codes J10, J11) were identified between July 1, 2011 and September 30, 2015. The date of the first observed influenza diagnosis during this period was designated as the index date. Patients were required to be aged at least 13 years at the time of diagnosis, with continuous medical and pharmacy benefits for at least 6 months prior to the index date (baseline period) and at least 3 months after the index date (follow-up period). Patients who died during the follow-up period were also included in the study. Patients were excluded if they had an influenza diagnosis during the baseline period.

Patients were categorized as initially hospitalized if they were diagnosed with influenza during hospitalization or if they were hospitalized within 0-1 day of the influenza diagnosis. 
The hospital admission date was captured as the start of the index hospitalization.

Among patients who were initially hospitalized, participants were further identified as potential high-risk patients if they met one or more of the criteria in Supplementary Fig. 1age, diagnostic code, or medication prescription criterion based on the Centers for Disease Control and Prevention (CDC) guidelines-at any time during baseline through the diagnosis date $[27,28]$. All conditions were identified using ICD-9-CM and ICD-10-CM codes.

Among high-risk initially hospitalized patients, we additionally identified if patients received care in the general ward, intensive unit (ICU), or/and if they received invasive or noninvasive mechanical ventilation (MV) during the index hospitalization. Services received in the ICU were identified using hospital revenue codes 200-209. ICD-9-Procedure Classification System (PCS) and ICD-10-PCS codes were utilized to identify MV.

High-risk patients who were diagnosed in the outpatient setting but never hospitalized during the 3-month follow-up period were classified as non-hospitalized patients.

\section{Study Variables}

Baseline patient demographics including age, sex, US geographic region, and place of diagnosis (inpatient + emergency department [ED], outpatient, other setting) were captured at the time of diagnosis. Diagnosis setting included ED (diagnosed in ED or inpatient setting), physician office, or other Medicare settings. Baseline comorbidities were assessed for the 6-month baseline period for all patients. Comorbidities including asthma, cancer, chronic obstructive pulmonary disease (COPD), chronic systemic steroids, congenital heart disease, congestive heart failure (CHF), coronary artery disease (CAD), cystic fibrosis, depression, diabetes, high cholesterol, HIV, chronic kidney disease (CKD), solid organ transplant, hematopoietic stem cell transplant, stroke, systemic lupus erythematosus, and previous evidence of pneumonia were identified using ICD-9-CM or ICD-10-CM codes.
Complications, all-cause mortality, death during inpatient stay, and 30-day readmission rates were also examined. Complications of interest were also based on CDC guidelines $[29,30]$ and identified using ICD-9-CM and ICD-10-CM codes. They included acute kidney failure, sinusitis, encephalomyelitis, Guillain-Barré syndrome, ischemic heart disease, acute myocarditis, myositis, pneumonia, respiratory failure, rhabdomyolysis, sepsis, stroke, otitis, upper and lower respiratory tract infection, upper respiratory tract infection, and lower respiratory tract infection. The proportion of patients who were discharged to an SNF and the length of stay (LOS) in an SNF were also evaluated. All-cause healthcare utilization and costs during 3 months pre- and 3 months postindex period were assessed, including hospital LOS across all hospitalizations; office visits; pharmacy use; and ED, inpatient, and outpatient visits. Costs were adjusted to 2015 US dollars using the medical care component of the Consumer Price Index.

\section{Statistical Methods}

All baseline and outcome variables were analyzed descriptively. Percentages and counts were provided for categorical variables. Means and standard deviations (SDs) were computed for continuous variables. Healthcare costs and utilizations were analyzed descriptively and compared between 3 months pre- and 3 months post-influenza diagnosis. Bivariate comparisons between pre- and post-index date periods were made using paired $t$ tests and McNemar's tests to test the statistical significance for continuous and categorical variables, respectively.

Multivariate logistic regression was conducted to examine potential predictors of initial hospitalization among high-risk hospitalized patients. All independent variables with $p<0.25$ on bivariate testing were initially included in the model. Variables with $p>0.10$ were dropped from the model sequentially unless they were identified as confounders (i.e., variables that, when dropped from the model, resulted in at least a $20 \%$ change in parameter estimates for one or more of the other variables 


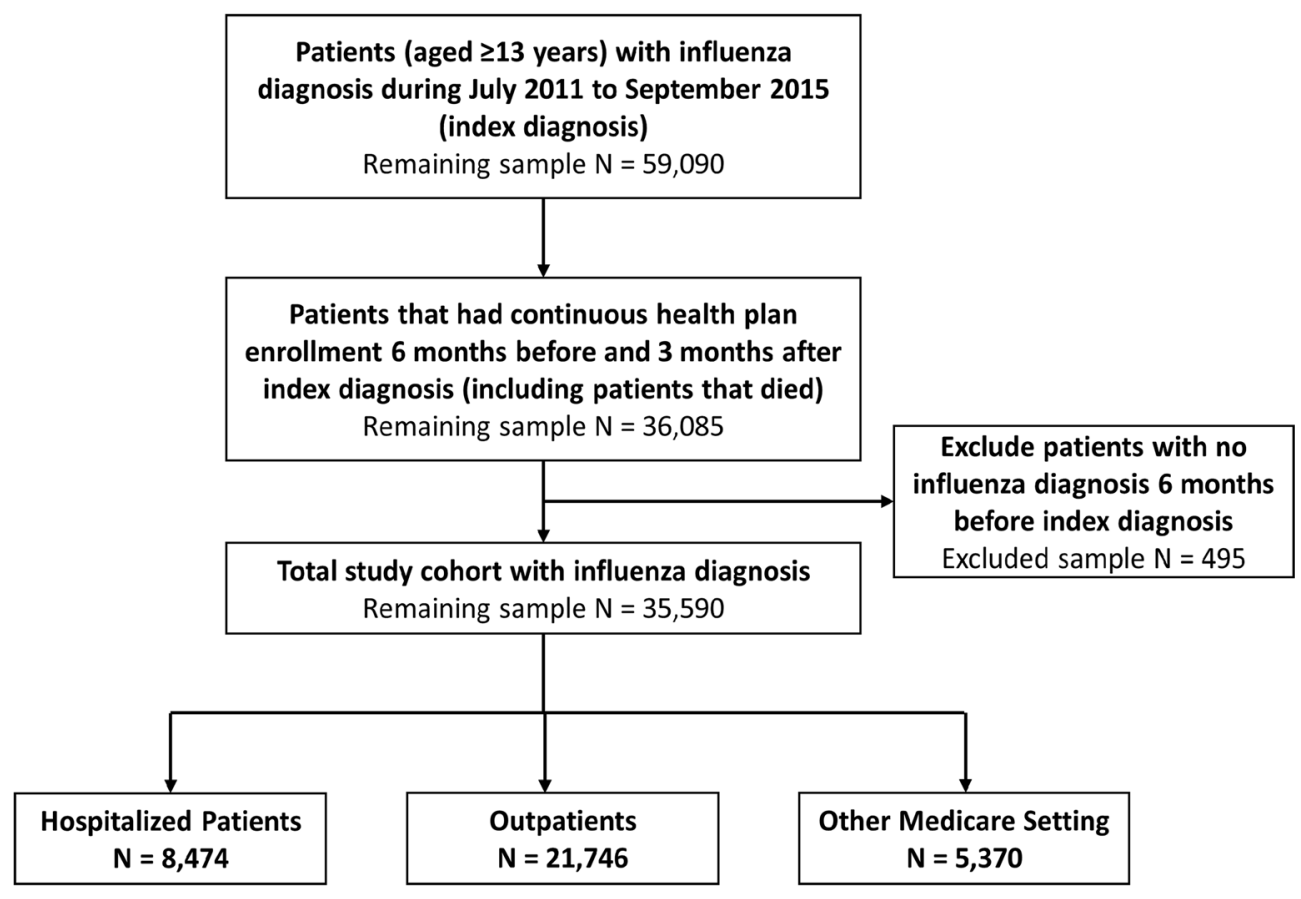

Fig. 1 Flowchart for patient inclusion criteria

when compared to the original model). The final model included confounders and all variables with $p<0.10$. Conditions defined as high risk based on CDC guidelines were initially included in the model. Comorbidities including congenital heart disease, cystic fibrosis, HIV, and ankylosing spondylitis were then removed from the model because they were prevalent in less than $1 \%$ of the population. Covariates in the final model included demographics, comorbidities, number of conditions, number of inpatient visits, and number of ED visits. Statistical analyses were conducted using Statistical Analysis System (SAS) v.9.3. (Cary, NC, USA). The $p$ value level of significance was set at $\alpha$ level 0.05 .

\section{Statement of Ethics Compliance}

This retrospective database analysis did not involve the collection, use, or transmittal of individual identifiable data. All patient identifiers in the database have been fully encrypted; therefore, neither institutional review board approval nor consent was necessary for this study, as it was conducted in the USA with depersonalized claims data and does not meet criteria for studies with human participants. It is therefore exempt from approval per the provision for unidentifiable personal data in the Federal Policy for the Protection of Human Subjects (1991).

\section{RESULTS}

\section{Baseline Characteristics}

Figure 1 shows the selection criteria for patients with influenza. A total of 59,090 patients aged at least 13 years and diagnosed with influenza were identified from the Medicare 5\% national sample administrative database. After applying the inclusion and exclusion criteria, the final analytical sample comprised a total of 35,590 patients including 8474 (23.8\%) patients who were initially hospitalized (within 1 day of influenza diagnosis), 21,746 (61.1\%) who were diagnosed in the outpatient setting, and 5370 (15.1\%) who were diagnosed in other Medicare settings. The study population included 8127 high-risk patients who were initially 
Table 1 Descriptive baseline for patients with influenza (6 months pre-diagnosis date)

\begin{tabular}{|c|c|c|c|c|c|c|}
\hline & \multicolumn{5}{|c|}{ Hospitalized patients $(N=8474)$} & \multirow{2}{*}{$\begin{array}{l}\text { Non-hospitalized } \\
(N=16,784)\end{array}$} \\
\hline & $\begin{array}{l}\text { All hospitalized } \\
(N=8474)\end{array}$ & $\begin{array}{l}\text { High-risk }^{\mathrm{a}} \\
(N=8127)\end{array}$ & $\begin{array}{l}\text { General ward }^{\mathrm{b}} \\
(N=5129)\end{array}$ & $\begin{array}{l}\mathrm{ICU}^{\mathrm{c}} \\
(N=2547)\end{array}$ & $\begin{array}{l}\mathrm{MV}^{\mathrm{d}} \\
(N=961)\end{array}$ & \\
\hline Age, mean (SD) & $76.2(14.1)$ & $77.3(13.3)$ & $76.8(14.3)$ & $74.8(13.9)$ & $\begin{array}{l}73.4 \\
\quad(13.3)\end{array}$ & $72.7(11.9)$ \\
\hline \multicolumn{7}{|l|}{ Sex, $n(\%)$} \\
\hline Male & $3139(37.0 \%)$ & $2970(36.5 \%)$ & $1812(35.3 \%)$ & $\begin{array}{l}1046 \\
(41.1 \%)\end{array}$ & $\begin{array}{l}394 \\
\quad(41.0 \%)\end{array}$ & $5736(34.2 \%)$ \\
\hline Female & $5335(63.0 \%)$ & $5157(63.5 \%)$ & $3317(64.7 \%)$ & $\begin{array}{l}1501 \\
(58.9 \%)\end{array}$ & $\begin{array}{l}567 \\
(59.0 \%)\end{array}$ & $11,048(65.8 \%)$ \\
\hline \multicolumn{7}{|l|}{ US geographic region, $n(\%)$} \\
\hline Northeast & $2021(23.8 \%)$ & $1958(24.1 \%)$ & $1346(26.2 \%)$ & $\begin{array}{l}472 \\
\quad(18.5 \%)\end{array}$ & $\begin{array}{l}231 \\
\quad(24.0 \%)\end{array}$ & $2818(16.8 \%)$ \\
\hline North Central & $2306(27.2 \%)$ & $2208(27.2 \%)$ & $1394(27.2 \%)$ & $\begin{array}{l}705 \\
\quad(27.7 \%)\end{array}$ & $\begin{array}{l}241 \\
\quad(25.1 \%)\end{array}$ & $3089(18.4 \%)$ \\
\hline South & $3015(35.6 \%)$ & $2866(35.3 \%)$ & $1806(35.2 \%)$ & $\begin{array}{l}960 \\
(37.7 \%)\end{array}$ & $\begin{array}{l}367 \\
\quad(38.2 \%)\end{array}$ & $8572(51.1 \%)$ \\
\hline West & $1118(13.2 \%)$ & $1081(13.3 \%)$ & $574(11.2 \%)$ & $\begin{array}{l}406 \\
\quad(15.9 \%)\end{array}$ & $\begin{array}{l}122 \\
(12.7 \%)\end{array}$ & $2205(13.1 \%)$ \\
\hline Unknown & $14(0.2 \%)$ & $14(0.2 \%)$ & $9(0.2 \%)$ & $4(0.2 \%)$ & $0(0.0 \%)$ & $100(0.6 \%)$ \\
\hline \multicolumn{7}{|l|}{ Comorbidities, $n(\%)$} \\
\hline Asthma & $1222(14.4 \%)$ & $1222(15.0 \%)$ & $736(14.3 \%)$ & $\begin{array}{l}363 \\
(14.3 \%)\end{array}$ & $\begin{array}{l}161 \\
(16.8 \%)\end{array}$ & $1731(10.3 \%)$ \\
\hline Cancer & $1007(11.9 \%)$ & $1007(12.4 \%)$ & $611(11.9 \%)$ & $\begin{array}{l}300 \\
(11.8 \%)\end{array}$ & $\begin{array}{l}106 \\
(11.0 \%)\end{array}$ & $1690(10.1 \%)$ \\
\hline Chronic obstructive pulmonary disease & $3325(39.2 \%)$ & $3246(39.9 \%)$ & $1858(36.2 \%)$ & $\begin{array}{l}1132 \\
(44.4 \%)\end{array}$ & $\begin{array}{l}505 \\
\quad(52.5 \%)\end{array}$ & $3579(21.3 \%)$ \\
\hline Chronic systemic steroids & $1495(17.6 \%)$ & $1447(17.8 \%)$ & $918(17.9 \%)$ & $\begin{array}{l}453 \\
\quad(17.8 \%)\end{array}$ & $\begin{array}{l}142 \\
(14.8 \%)\end{array}$ & $3311(19.7 \%)$ \\
\hline Congenital heart disease & $65(0.8 \%)$ & $65(0.8 \%)$ & $39(0.8 \%)$ & $19(0.7 \%)$ & $11(1.1 \%)$ & $73(0.4 \%)$ \\
\hline Congestive heart failure & $2357(27.8 \%)$ & $2357(29.0 \%)$ & $1285(25.1 \%)$ & $\begin{array}{l}818 \\
\quad(32.1 \%)\end{array}$ & $\begin{array}{l}359 \\
(37.4 \%)\end{array}$ & $1530(9.1 \%)$ \\
\hline Coronary artery disease & $2447(28.9 \%)$ & $2447(30.1 \%)$ & $1387(27.0 \%)$ & $\begin{array}{l}793 \\
(31.1 \%)\end{array}$ & $\begin{array}{l}324 \\
(33.7 \%)\end{array}$ & $3046(18.1 \%)$ \\
\hline Cystic fibrosis & $15(0.2 \%)$ & $15(0.2 \%)$ & $10(0.2 \%)$ & $4(0.2 \%)$ & $2(0.2 \%)$ & $9(0.1 \%)$ \\
\hline Depression & $1574(18.6 \%)$ & $1502(18.5 \%)$ & $943(18.4 \%)$ & $\begin{array}{l}488 \\
\quad(19.2 \%)\end{array}$ & $\begin{array}{l}204 \\
\quad(21.2 \%)\end{array}$ & $1768(10.5 \%)$ \\
\hline Diabetes & $3336(39.4 \%)$ & $3243(39.9 \%)$ & $1924(37.5 \%)$ & $\begin{array}{l}1090 \\
\quad(42.8 \%)\end{array}$ & $\begin{array}{l}437 \\
\quad(45.5 \%)\end{array}$ & $4979(29.7 \%)$ \\
\hline High cholesterol & $3776(44.6 \%)$ & $3679(45.3 \%)$ & $2216(43.2 \%)$ & $\begin{array}{l}1185 \\
(46.5 \%)\end{array}$ & $\begin{array}{l}428 \\
(44.5 \%)\end{array}$ & $7421(44.2 \%)$ \\
\hline Human immunodeficiency virus & $74(0.9 \%)$ & $74(0.9 \%)$ & $50(1.0 \%)$ & $20(0.8 \%)$ & $4(0.4 \%)$ & $113(0.7 \%)$ \\
\hline Chronic liver disease & $168(2.0 \%)$ & $168(2.1 \%)$ & $101(2.0 \%)$ & $53(2.1 \%)$ & $17(1.8 \%)$ & $323(1.9 \%)$ \\
\hline Chronic kidney disease & $1859(21.9 \%)$ & $1859(22.9 \%)$ & $992(19.3 \%)$ & $\begin{array}{l}674 \\
\quad(26.5 \%)\end{array}$ & $\begin{array}{l}252 \\
\quad(26.2 \%)\end{array}$ & $1394(8.3 \%)$ \\
\hline Solid organ transplant & $213(2.5 \%)$ & $208(2.6 \%)$ & $128(2.5 \%)$ & $72(2.8 \%)$ & $25(2.6 \%)$ & $163(1.0 \%)$ \\
\hline Stem cell transplant & $29(0.3 \%)$ & $29(0.4 \%)$ & $12(0.2 \%)$ & $14(0.5 \%)$ & $8(0.8 \%)$ & $28(0.2 \%)$ \\
\hline Stroke & $551(6.5 \%)$ & $551(6.8 \%)$ & $317(6.2 \%)$ & $181(7.1 \%)$ & $74(7.7 \%)$ & $492(2.9 \%)$ \\
\hline Systemic lupus erythematosus & $119(1.4 \%)$ & $116(1.4 \%)$ & $67(1.3 \%)$ & $42(1.6 \%)$ & $17(1.8 \%)$ & $177(1.1 \%)$ \\
\hline Previous evidence of pneumonia, $n(\%)$ & $1336(15.8 \%)$ & $1306(16.1 \%)$ & $715(13.9 \%)$ & $\begin{array}{l}506 \\
\quad(19.9 \%)\end{array}$ & $\begin{array}{l}241 \\
\quad(25.1 \%)\end{array}$ & $846(5.0 \%)$ \\
\hline
\end{tabular}


Table 1 continued

\begin{tabular}{|c|c|c|c|c|c|c|}
\hline & \multicolumn{5}{|c|}{ Hospitalized patients $(N=8474)$} & \multirow{2}{*}{$\begin{array}{l}\text { Non-hospitalized } \\
(N=16,784)\end{array}$} \\
\hline & $\begin{array}{l}\text { All hospitalized } \\
(N=8474)\end{array}$ & $\begin{array}{l}\text { High-risk }^{\mathbf{a}} \\
(N=8127)\end{array}$ & $\begin{array}{l}\text { General ward }^{\mathbf{b}} \\
(N=5129)\end{array}$ & $\begin{array}{l}\text { ICU }^{c} \\
(N=2547)\end{array}$ & $\begin{array}{l}M V^{\mathrm{d}} \\
(N=961)\end{array}$ & \\
\hline $\begin{array}{l}\text { Patients with influenza vaccination } \\
\text { during baseline period, } n(\%)\end{array}$ & $3941(46.5 \%)$ & $3850(47.4 \%)$ & $2433(47.4 \%)$ & $\begin{array}{l}1140 \\
(44.8 \%)\end{array}$ & $\begin{array}{l}412 \\
(42.9 \%)\end{array}$ & $8091(48.2 \%)$ \\
\hline \multicolumn{7}{|l|}{ Place of diagnosis, $n(\%)$} \\
\hline Inpatient setting $+\mathrm{ED}$ & $7577(89.4 \%)$ & $7261(89.3 \%)$ & $4579(89.3 \%)$ & $\begin{array}{l}2273 \\
\quad(89.2 \%)\end{array}$ & $\begin{array}{l}868 \\
(90.3 \%)\end{array}$ & $4471(26.6 \%)$ \\
\hline Outpatient setting & $625(7.4 \%)$ & $608(7.5 \%)$ & $420(8.2 \%)$ & $155(6.1 \%)$ & $52(5.4 \%)$ & $12,313(73.4 \%)$ \\
\hline Other settings & $272(3.2 \%)$ & $258(3.2 \%)$ & $130(2.5 \%)$ & $119(4.7 \%)$ & $41(4.3 \%)$ & - \\
\hline
\end{tabular}

$E D$ emergency department, $I C U$ intensive care unit, $M V$ mechanical ventilation, $S D$ standard deviation

a Patients were identified as high risk if they were aged 65+ years at the time of influenza diagnosis, had a diagnostic code for chronic lung disease (asthma, chronic obstructive pulmonary disease, or cystic fibrosis), cardiovascular or cerebrovascular conditions (congenital heart disease, congestive heart failure, coronary artery disease, or stroke), and a weakened immune system (HIV, cancer, chronic liver or kidney disease, or chronic systemic steroids) during the baseline period or on the diagnosis date

b Patients that did not use services in the ICU or MV during the index hospitalization

${ }^{c}$ Patients that used services in the ICU during index hospitalization. ICU and MV are not mutually exclusive

${ }^{\mathrm{d}}$ Patients that used MV during index hospitalization. ICU and MV are not mutually exclusive

hospitalized and 16,784 who were not hospitalized. Among patients who were initially hospitalized with influenza, 5129 (60.5\%) were admitted in the general ward, $2547(30.1 \%)$ received care in the ICU, and 961 (11.3\%) patients were administered MV during their initial hospitalization.

Patient characteristics and place of diagnosis are summarized in Table 1. Among hospitalized patients, $89.4 \%$ were diagnosed in the $\mathrm{ED}$, whereas $7.4 \%$ and $3.2 \%$ were diagnosed in a physician's office or other Medicare settings, respectively. On average, hospitalized patients were older (76 years) than those who were not (73 years). The total sample population included preponderances of women $(63.0 \%)$ and residents of the South US region (35.6\%). More than $45 \%$ of the patients had an influenza vaccine during the baseline period. Of high-risk patients initially hospitalized, high cholesterol (45.3\%), COPD (39.9\%), diabetes (39.9\%), CAD (30.1\%), CHF (29\%), and CKD (22.9\%) were among the most prevalent comorbid conditions during the baseline period. These conditions were also the most common comorbid conditions among patients in the general ward, ICU, on $\mathrm{MV}$, or among those who were not hospitalized.

\section{Predictors of Initial Hospitalization}

Figure 2 shows predictors of initial hospitalization using a logistic regression model. Comorbidities such as diabetes (odds ratio [OR] 1.25; $p<0.001$ ), COPD (OR 2.05; $p<0.001)$, CHF (OR 1.76; $p<0.001)$, stroke (OR 1.19; $p<0.001$ ), CKD (OR 1.60; $p<0.001$ ), solid organ transplant (OR 1.92; $p<0.001$ ), previous evidence of pneumonia (OR 1.26; $p<0.001$ ), older age (at least 75 years), number of conditions (at least two), and baseline healthcare resource use (at least one inpatient or ER visit) were statistically significant $(p<0.05)$ predictors of initial hospitalization after adjusting for other covariates. Being female (OR 0.82; $p<0.001)$, having comorbidities such as asthma (OR $0.71 ; p<0.05)$, chronic liver disease (OR 076; $p=0.016$ ), osteoarthritis (OR $0.89 ; p=0.007)$ were associated with lower risk of hospitalization.

\section{Outcome Assessment}

Table 2 illustrates complications of patients with influenza during the follow-up period. During the 90-day post-influenza diagnosis, upper and lower respiratory tract infections 


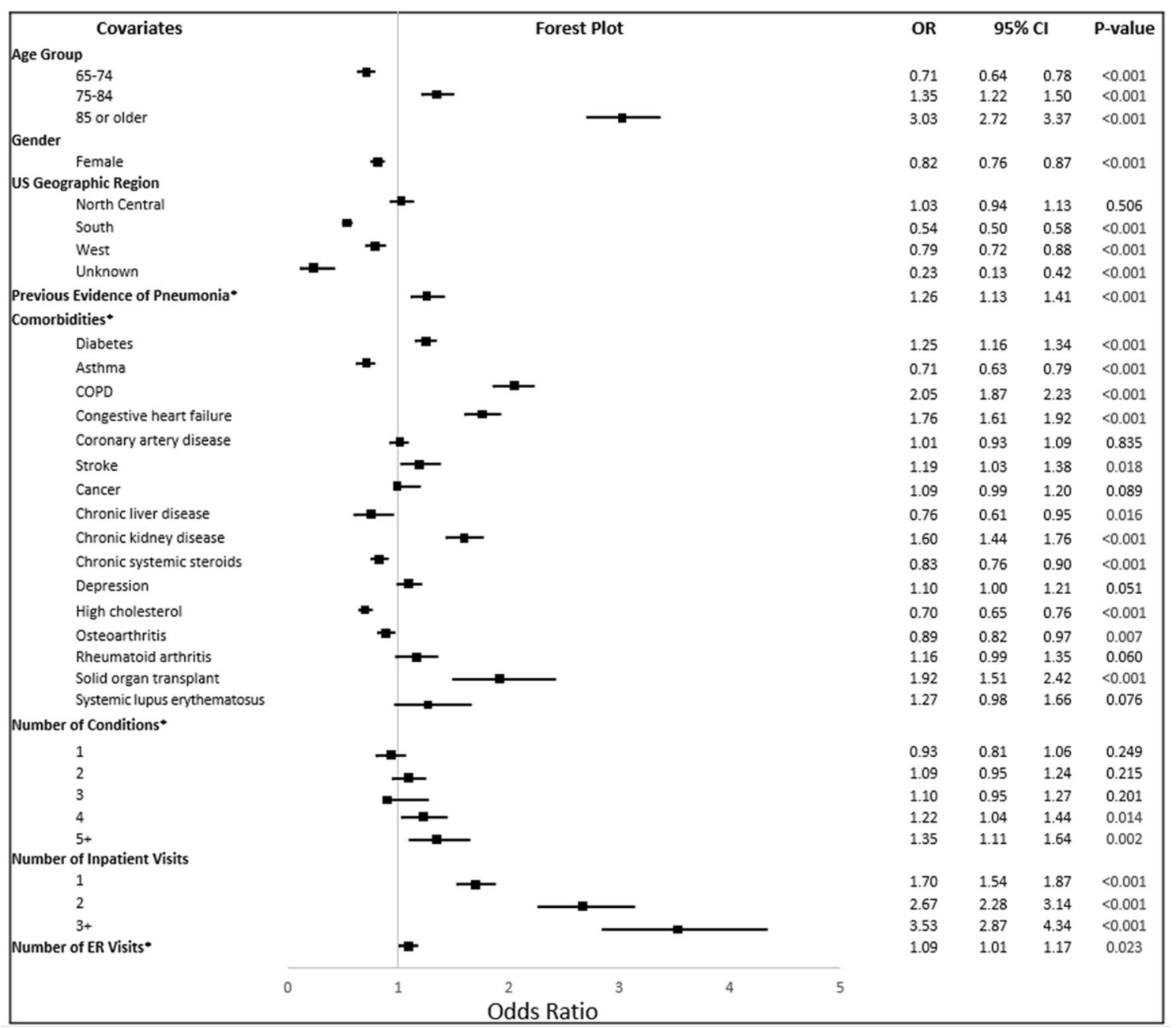

Fig. 2 Predictors of initial hospitalization among patients with influenza. OR $>1$ indicates that the variable is a positive predictor of hospitalization among patients that were initially hospitalized $(N=8474)$ compared to patients that were never hospitalized $(N=16,784)$. The following were used as references: age (less than 65 years), gender (male), US geographic region (northeast), number of comorbidities $(n=0)$, number of inpatient visits $(n=0)$. CI confidence interval, COPD chronic

$(62.9 \%)$, previous evidence of pneumonia (59.0\%), and ischemic heart disease (44.4\%) were predominant complications among highrisk patients initially hospitalized. These conditions were also the most common complications among hospitalized patients in the general ward, ICU, on MV (particularly among patients administered $\mathrm{MV}$ in their initial obstructive pulmonary disease, ER emergency room, OR odds ratio. *Variables such as previous evidence of pneumonia, comorbidities, number of conditions, healthcare utilization (including number of inpatient ER visits) were evaluated anytime during the baseline period were evaluated. The other variables such as age, gender, and US geographic region were evaluated on the index date

hospitalization). The complications rates were much lower among patients who were not hospitalized.

Among high-risk patients initially hospitalized, the all-cause mortality rate was $5.1 \%$ during inpatient stays and $9.3 \%$ within 30 days of influenza diagnosis (Table 3). Among high-risk patients discharged from the hospital, 19.6\% 
Table 2 Complication among patients with influenza during the follow-up period

\begin{tabular}{|c|c|c|c|c|c|c|}
\hline & \multicolumn{5}{|c|}{ Hospitalized patients } & \multirow{2}{*}{$\begin{array}{l}\text { Non- } \\
\text { hospitalized } \\
(N=16,784)\end{array}$} \\
\hline & $\begin{array}{l}\text { All hospitalized } \\
(N=8474)\end{array}$ & $\begin{array}{l}\text { High-risk }^{\mathrm{a}} \\
(N=8127)\end{array}$ & $\begin{array}{l}\text { General ward }^{\mathrm{b}} \\
(N=5129)\end{array}$ & $\begin{array}{l}\mathrm{ICU}^{\mathrm{c}} \\
(N=2547)\end{array}$ & $\begin{array}{l}M V^{\mathrm{d}} \\
(N=961)\end{array}$ & \\
\hline \multicolumn{7}{|l|}{ Complications, $n(\%)$} \\
\hline Acute kidney failure & $2212(26.1 \%)$ & $2165(26.6 \%)$ & $1075(21.0 \%)$ & $916(36.0 \%)$ & $439(45.7 \%)$ & $155(0.9 \%)$ \\
\hline Sinusitis & $378(4.5 \%)$ & $350(4.3 \%)$ & $245(4.8 \%)$ & $103(4.0 \%)$ & $37(3.9 \%)$ & $1400(8.3 \%)$ \\
\hline Encephalomyelitis & $11(0.1 \%)$ & $9(0.1 \%)$ & $6(0.1 \%)$ & $1(0.0 \%)$ & $3(0.3 \%)$ & $4(0.0 \%)$ \\
\hline Guillain-Barré syndrome & $458(5.4 \%)$ & $445(5.5 \%)$ & $268(5.2 \%)$ & $153(6.0 \%)$ & $48(5.0 \%)$ & $171(1.0 \%)$ \\
\hline Ischemic heart disease & $3625(42.8 \%)$ & $3609(44.4 \%)$ & $2027(39.5 \%)$ & $1221(47.9 \%)$ & $485(50.5 \%)$ & $2273(13.5 \%)$ \\
\hline Kidney failure & $2327(27.5 \%)$ & $2278(28.0 \%)$ & $1143(22.3 \%)$ & $955(37.5 \%)$ & $451(46.9 \%)$ & $221(1.3 \%)$ \\
\hline Acute myocarditis & $5(0.1 \%)$ & $5(0.1 \%)$ & $3(0.1 \%)$ & $2(0.1 \%)$ & $0(0.0 \%)$ & $1(0.0 \%)$ \\
\hline Myositis & $325(3.8 \%)$ & $300(3.7 \%)$ & $199(3.9 \%)$ & $94(3.7 \%)$ & $39(4.1 \%)$ & $599(3.6 \%)$ \\
\hline Pneumonia & $4977(58.7 \%)$ & $4796(59.0 \%)$ & $2805(54.7 \%)$ & $1742(68.4 \%)$ & $775(80.6 \%)$ & $1647(9.8 \%)$ \\
\hline Respiratory failure & $2552(30.1 \%)$ & $2460(30.3 \%)$ & $909(17.7 \%)$ & $1344(52.8 \%)$ & $864(89.9 \%)$ & $66(0.4 \%)$ \\
\hline Rhabdomyolysis & $163(1.9 \%)$ & $157(1.9 \%)$ & $81(1.6 \%)$ & $63(2.5 \%)$ & $23(2.4 \%)$ & $14(0.1 \%)$ \\
\hline Sepsis & $1279(15.1 \%)$ & $1209(14.9 \%)$ & $682(13.3 \%)$ & $486(19.1 \%)$ & $174(18.1 \%)$ & $53(0.3 \%)$ \\
\hline Stroke & $574(6.8 \%)$ & $570(7.0 \%)$ & $294(5.7 \%)$ & $229(9.0 \%)$ & $73(7.6 \%)$ & $227(1.4 \%)$ \\
\hline Otitis & $80(0.9 \%)$ & $76(0.9 \%)$ & $56(1.1 \%)$ & $16(0.6 \%)$ & $5(0.5 \%)$ & $316(1.9 \%)$ \\
\hline $\begin{array}{l}\text { Upper and lower respiratory } \\
\text { tract infection }\end{array}$ & $5328(62.9 \%)$ & $5111(62.9 \%)$ & $3124(60.9 \%)$ & $1750(68.7 \%)$ & $724(75.3 \%)$ & $6055(36.1 \%)$ \\
\hline
\end{tabular}

ICU intensive care unit, $M V$ mechanical ventilation

a Patients were identified as high risk if they were aged $65+$ years at the time of influenza diagnosis, had a diagnostic code for chronic lung disease (asthma, chronic obstructive pulmonary disease, or cystic fibrosis), cardiovascular or cerebrovascular conditions (congenital heart disease, congestive heart failure, coronary artery disease, or stroke), and a weakened immune system (HIV, cancer, chronic liver or kidney disease, or chronic systemic steroids) during the baseline period or on the diagnosis date

b Patients that did not use services in the ICU or MV during the index hospitalization

c Patients that used services in the ICU during index hospitalization. ICU and MV are not mutually exclusive

d Patients that used MV during index hospitalization. ICU and MV are not mutually exclusive

were discharged to an SNF; 76.4\% of these were not in an SNF prior to hospitalization. The 30 -day readmission rate was $14.0 \%$, and the allcause mortality rate within 30 days of influenza diagnosis was $29.8 \%$ among MV patients, $15.6 \%$ among ICU patients, and $0.8 \%$ among patients who were not hospitalized (Table 3 ).

\section{Pre-Post Analysis}

Table 4 illustrates pre-post analysis of healthcare resource utilization and costs among hospitalized and non-hospitalized patients. Among all hospitalized patients, there were significant increases in the number of patients who had one inpatient admission (from $15.6 \%$ to $71.3 \%$; $p<0.001$ ), two inpatient admissions (from
$4.9 \%$ to $18.55 ; p<0.001)$, and three or more inpatient admissions (from $2.75 \%$ to $8.5 \%$ ) after influenza diagnosis. In addition, there were increases in the number of inpatient visits (from 0.3 to 1.4 visits; $p<0.001$ ), number of outpatient visits (from 7.8 visits to 8.2 visits; $p<0.001$ ), and longer LOS across all hospitalizations (from 2.1 to 8.7 days; $p<0.001$ ), whereas the number of pharmacy visits decreased from 9.6 to $8.0(p<0.001)$ after influenza diagnosis. Total average all-cause healthcare costs among all hospitalized patients were increased by $\$ 16,568$ per patient $(p<0.001)$ predominantly as a result of increased all-cause hospitalization costs (from $\$ 4305$ to $\$ 16,651 ; p<0.001)$. 
Table 3 Descriptive outcomes for patients with influenza

\begin{tabular}{|c|c|c|c|c|c|}
\hline & \multicolumn{5}{|c|}{ Hospitalized patients $(N=8474)$} \\
\hline & $\begin{array}{l}\text { All hospitalized } \\
(N=8474)\end{array}$ & $\begin{array}{l}\text { High-risk }^{\mathrm{a}} \\
(N=8127)\end{array}$ & $\begin{array}{l}\text { General ward }^{\mathrm{b}} \\
(N=5129)\end{array}$ & $\begin{array}{l}\mathrm{ICU}^{\mathrm{c}} \\
(N=2547)\end{array}$ & $\begin{array}{l}M^{d} \\
(N=961)\end{array}$ \\
\hline $\begin{array}{l}\text { Patients who were discharged to SNF, } \\
n(\%)\end{array}$ & $1606(19.0 \%)$ & $1590(19.6 \%)$ & $889(17.3 \%)$ & $564(22.1 \%)$ & $227(23.6 \%)$ \\
\hline $\begin{array}{l}\% \text { of patients not in SNF prior to } \\
\text { hospitalization }\end{array}$ & $1231(76.7 \%)$ & $1215(76.4 \%)$ & $692(77.8 \%)$ & $431(76.4 \%)$ & $173(76.2 \%)$ \\
\hline $\begin{array}{l}\% \text { of patients in SNF prior to } \\
\text { hospitalization }\end{array}$ & $375(23.3 \%)$ & $375(23.6 \%)$ & $197(22.2 \%)$ & $133(23.6 \%)$ & $54(23.8 \%)$ \\
\hline Length of stay in skilled nursing facility & $6.4(31.4)$ & $6.6(31.9)$ & $6.1(31.5)$ & $7.2(33.5)$ & $7.7(25.4)$ \\
\hline \multicolumn{6}{|l|}{ All-cause mortality, $n(\%)$} \\
\hline 30-day & $764(9.0 \%)$ & $752(9.3 \%)$ & $264(5.1 \%)$ & $397(15.6 \%)$ & $286(29.8 \%)$ \\
\hline 90-day & $1186(14.0 \%)$ & $1168(14.4 \%)$ & $476(9.3 \%)$ & $565(22.2 \%)$ & $364(37.9 \%)$ \\
\hline \multicolumn{6}{|l|}{ Death during inpatient stay, $n(\%)$} \\
\hline 30-day & $426(5.0 \%)$ & $417(5.1 \%)$ & $97(1.9 \%)$ & $275(10.8 \%)$ & $222(23.1 \%)$ \\
\hline 90-day & $565(6.7 \%)$ & $553(6.8 \%)$ & $154(3.0 \%)$ & $345(13.5 \%)$ & $270(28.1 \%)$ \\
\hline 30-day readmission, $n$ (\%) & $1167(13.8 \%)$ & $1134(14.0 \%)$ & $660(12.9 \%)$ & $398(15.6 \%)$ & $142(14.8 \%)$ \\
\hline $\begin{array}{l}\text { ICU intensive care unit, } M V \text { mechanical } \\
\text { a Patients were identified as high risk if they } \\
\text { chronic obstructive pulmonary disease, or } \\
\text { coronary artery disease, or stroke), and a we } \\
\text { baseline period or on the diagnosis date } \\
\text { b Patients that did not use services in the } \\
{ }^{\mathrm{c}} \text { Patients that used services in the ICU du } \\
\text { d Patients that used MV during index hos }\end{array}$ & $\begin{array}{l}\text { ntilation, } S N F \text { skill } \\
\text { were aged } 65+\text { yea } \\
\text { ystic fibrosis), cardi } \\
\text { akened immune sys } \\
\text { CU or MV during } \\
\text { ring index hospitali }\end{array}$ & $\begin{array}{l}\text { ng facility } \\
\text { time of influenza } \\
\mathrm{r} \text { or cerebrovascu } \\
\mathrm{V} \text {, cancer, chroni } \\
\text { ex hospitalization } \\
\text { ICU and MV are }\end{array}$ & $\begin{array}{l}\text { osis, had a diagnos } \\
\text { nditions (congenit } \\
\text { r or kidney disease, } \\
\text { mutually exclusive }\end{array}$ & $\begin{array}{l}\text { for chronic lur } \\
\text { disease, conge } \\
\text { onic systemic st }\end{array}$ & $\begin{array}{l}\text { lisease (asthma, } \\
\text { e heart failure, } \\
\text { ids) during the }\end{array}$ \\
\hline
\end{tabular}

\section{DISCUSSION}

This real-world retrospective study of the Medicare 5\% national sample administrative database described patient characteristics, identified predictors of initial hospitalization, and described the current outcomes of high-risk patients hospitalized with influenza. The study population included preponderances of women and residents of the South US geographic region, which generally align with the predominantly female Medicare population as well as geographic patterns of reported influenza cases as of 2019 [29, 31]. Of high-risk patients initially hospitalized, COPD, CHF, and CKD were among the strongest predictors of hospitalization-in line with CDC definitions. Other significant predictors included older age, being male, greater comorbidity burden, and higher baseline healthcare resource use. All-cause mortality was $5.1 \%$ during inpatient stayssomewhat higher than the $3.6 \%$ reported for all flu cases in the 2015-2016 season [30]. Total costs associated with the index hospitalization represented considerable economic burden, with an average of $\$ 16,568$ per patient.

The influenza virus leads to significant morbidity and mortality each year in the USA, with 36,000 reported deaths, more than 226,000 hospitalizations, approximately 3.1 million hospitalization days, and costs of $\$ 5$ billion associated with these hospitalizations annually $[32,33]$. Although in most cases influenza is a self-limiting disease from which patients can recover without the risk of serious complications $[6,7]$, the burden of disease among hospitalized patients remains high. Hospitalization is generally frequent among very young and elderly patients and especially among those with underlying high-risk medical conditions including chronic lung disease, cardiovascular or cerebrovascular disease, and weakened immune system $[19,34]$. Severe or complicated influenza is associated with lower respiratory tract infection (hypoxemia, shortness of breath, lung infiltrate), central nervous system 
Table 4 Pre-post analysis of healthcare utilization and costs among patients with influenza

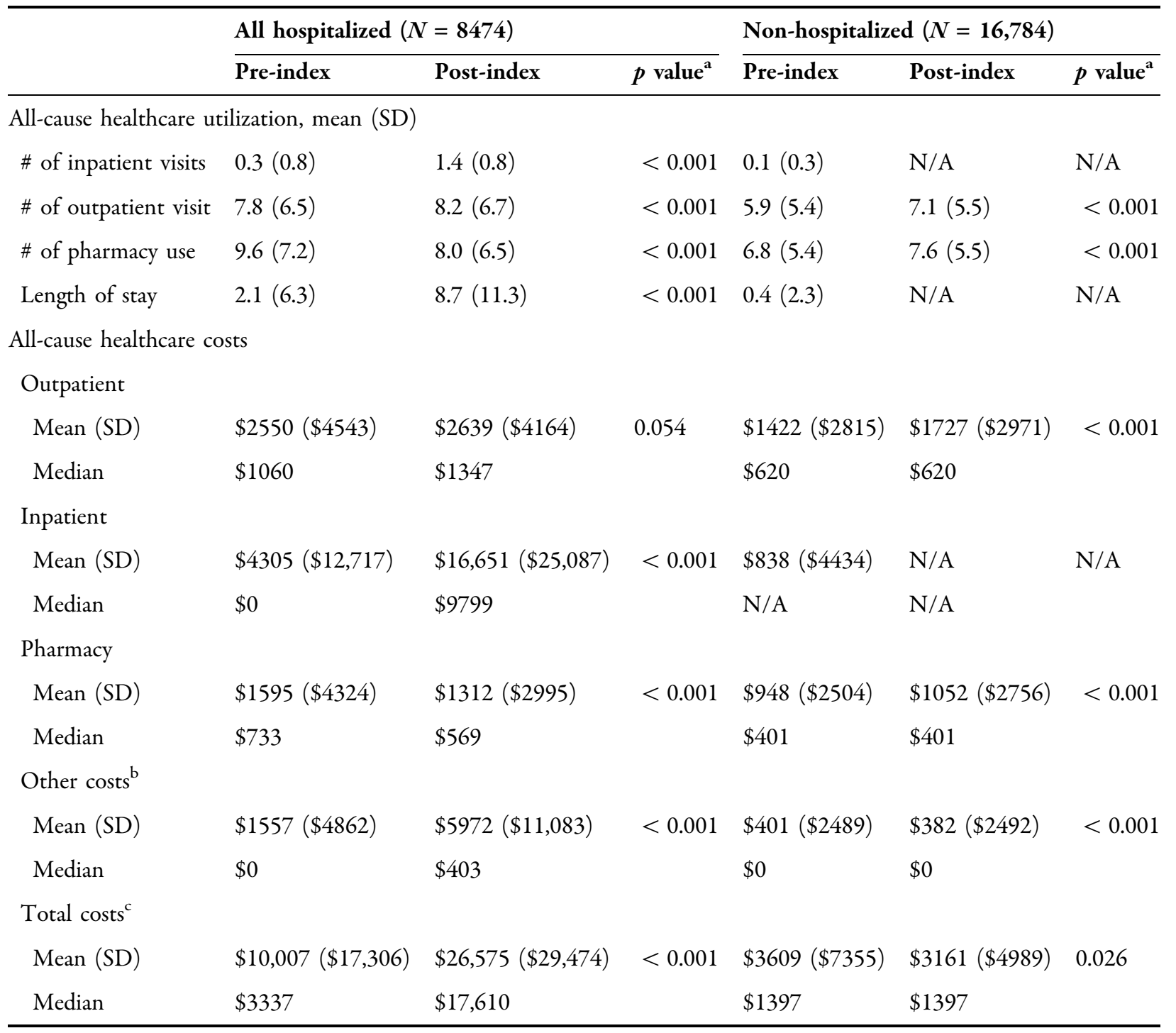

$N / A$ not applicable, $S D$ standard deviation

${ }^{a} p$ values were calculated using Wilcoxon rank-sum tests

b Other costs includes durable medical equipment (DME), home health agency (HHA), hospice, and skilled nursing facility (SNF)

${ }^{c}$ Total costs consist of the sum of inpatient costs. Outpatient costs, pharmacy costs, and other costs

involvement, and/or a significant exacerbation of an underlying medical condition which requires hospitalization and ICU with or without MV and may result in death among highrisk patients [8, 35-37]. Pneumonia, bronchitis, croup, exacerbations of respiratory conditions (COPD, asthma), and cardiovascular complications (heart attack, pericarditis, myocarditis) are frequent complications of influenza [38, 39]. In our study, most admitted high-risk patients were older (average age 77 years), and many suffered from complications including upper and lower respiratory tract infections (62.9\%), pneumonia (59.0\%), and ischemic heart disease (44.4\%) during the 90 days post influenza diagnosis. A significantly higher proportion of 
patients had pneumonia in the post-index period compared to the pre-index period, which may be attributed to influenza-related secondary infections.

The burden of hospitalization among patients with influenza contributes the largest share of healthcare costs in the management of influenza. In the current analysis, there were significant increases in the number of medical resource utilizations after patients were diagnosed with influenza, including an increase in the number of inpatient admissions and longer hospital LOS. Total average all-cause healthcare costs among all admitted patients increased by $\$ 16,568$ per patient $(p<0.0001)$, predominantly as a result of increased all-cause hospitalization costs (from $\$ 4305$ to $\$ 16,651$; $p<0.0001$ ).

Analysis from a US managed care database indicated that the total healthcare costs (adjusted to 2015 dollars) of patients with influenza were \$2881. However, a significantly higher average total cost (\$3609) was observed among patients with complicated influenza, where inpatient costs (29\%) were the main cost drivers accounting for $35 \%$ of the total costs [5]. It is also important to note that managed care plans are capitated and may include Medicare/ Medicaid dual beneficiaries. As NAIs have been shown to reduce LOS and several specific inpatient cost drivers $[40,41]$, their use could have an important impact on economic outcomes.

ED visits, hospitalizations, ICU admissions, and deaths due to respiratory complications play an important role in the clinical burden of influenza. Patients aged 65 years or older are at a greater risk of hospitalization, pneumonia, heart attack, or stroke within 2 weeks following infection [42]. These patients are often discharged to long-term care facilities such as skilled nursing homes, rehabilitation centers, long-term care hospitals, and psychiatric care facilities, increasing the burden at these settings [43]. Overall, 5.1\% of the admitted patients died during inpatient stay and $9.2 \%$ died within 30 days of diagnosis. At discharge, $19 \%$ of the admitted patients in the current study were discharged to SNF where they remained on average for 6.4 days. Despite the substantial disease burden, influenza is often not recognized by admitting physicians who are forced to rely on symptomology rather than confirmed laboratory testing [44, 45], and influenza treatments may subsequently be delayed. To attain the best possible outcomes, it is important for physicians to quickly identify patients who need more intensive treatment. EDs are likely to be the first point of care for high-risk patients. In a recent retrospective analysis of laboratory-confirmed influenza cases in Serbia, Dimitrijević et al. reported that at age over 65 years, the presence of any comorbidity (including chronic respiratory diseases, asthma, diabetes, chronic heart disease, CKD, neurological disease, immunocompromised status) or at least two comorbidities were among predictors of hospitalization or admission to ICU [46]. In our study we found that predictors of hospitalization in this study aligned with the increased risk of complication and may help physicians to recognize the predictors for hospitalization. These predictors could be one of several considerations for ED physicians in whether to observe or admit patients rather than discharging them.

Influenza vaccine is effective only in about $40-60 \%$ of elderly patients [47]. Hence, antiviral treatments are required to attenuate the infection and reduce the risk of mortality in elderly populations. Uncomplicated influenza can typically be managed with bed rest and sufficient hydration, possibly supplemented by palliative use of over-the-counter pain and fever relievers, antihistamines, and decongestants [48], whereas complicated influenza and influenza in high-risk patients requires management with antiviral medications, mainly NAIs [6-8]. Oseltamivir, zanamivir, and peramivir have been approved for the treatment of uncomplicated influenza in the USA. However, extant literature is characterized by heterogeneity in study design, specific parameters, and populations, and there is little consensus regarding hospitalizations and complications for oseltamivir and zanamivir [49-52]. Resistance to NAIs has also been documented; as mentioned, the therapy is most effective if initiated within $48 \mathrm{~h}$ of symptom onset. It is imperative to effectively diagnose patients and administer antiviral treatment earlier in order to mitigate and 
reduce the risk of complications and economic burden among admitted patients with influenza.

\section{Limitations}

As with all retrospective claims analyses, this study is limited to the observation of associations rather than the inference of causality. Claims data are also subject to coding errors or incorrectly entered diagnoses that were primarily coded for reimbursement purposes rather than clinical accuracy. In this study, influenza diagnoses were captured on the basis of ICD-9/ICD-10 codes and not on the basis of clinical parameters. The presence of an ICD-9/ ICD-10 diagnosis code for influenza on a medical claim does not necessarily indicate the presence of influenza. There could be an inherent difficulty of distinguishing between influenza and influenza-like illness; additionally, as a result of the lack of laboratory values in the database, influenza diagnosis could not be confirmed. This may have led to over- or underestimation of influenza cases, and results should be interpreted accordingly. Complications were identified as conditions that occurred 3 months following an influenza diagnosis date using ICD-9/ICD-10 codes; therefore, we could not ascertain if the conditions were a real complication of influenza or an underlying disease that may have been exacerbated as a result of influenza disease. Complications of influenza related to the worsening or exacerbation of underlying chronic conditions such as asthma, COPD, diabetes, or CHF were not captured because of the lack in the Medicare database of diagnostic code associated with worsening of these chronic conditions. Therefore, the rate of complications might be underestimated in this population. This study included only fee-for-service Medicare enrollees, which may limit the generalizability of the study results to patients with managed care, commercial insurance plans, or some combination thereof. Moreover, the Medicare population includes preponderances of women and residents of the South US geographic region, which may further limit generalizability to broader populations. Finally, the dataset terminates in 2015 and therefore does not reflect more recent developments in the treatment landscape, such as the use of baloxavir marboxil in the past two influenza seasons.

\section{CONCLUSIONS}

The study confirms that older age; greater comorbidity burden; and diagnosis of COPD, $\mathrm{CHF}$, or CKD are predictors of hospitalization that can help identify high-risk patients with severe influenza. Moreover, the considerable clinical and economic burden associated with these risk factors together with the importance of timely diagnosis and treatment underscores the importance of early detection, especially as EDs are likely to be the first point of care for these patients. These data can be useful for researchers, physicians, and other stakeholders to help guide future influenza research, management, and prevention programs.

\section{ACKNOWLEDGEMENTS}

Funding. This work and the journal's Rapid Service Fees were supported by Janssen Global Services, LLC.

Medical Writing, Editorial or Other Assistance. Research assistance in the preparation of this article was provided by Janvi Sah of SIMR, LLC. Formatting and editing support was provided by Chris Haddlesey of SIMR, LLC.

Authorship. All named authors meet the International Committee of Medical Journal Editors (ICMJE) criteria for authorship for this article, take responsibility for the integrity of the work as a whole, and have given their approval for this version to be published.

Disclosures. Susan C. Bolge and Roman Fleischhackl are employees of Janssen, the study sponsor. Furaha Kariburyo is an employee of SIMR, LLC, a paid consultant to the study 
sponsor. Huseyin Yuce has no conflicts to declare.

Compliance with Ethical Guidelines. This retrospective database analysis did not involve the collection, use, or transmittal of individual identifiable data. All patient identifiers in the database have been fully encrypted; therefore neither institutional review board approval nor consent was necessary for this study, as it was conducted in the USA with depersonalized claims data and does not meet criteria for studies with human participants; it is therefore exempt from approval per the provision for unidentifiable personal data in the Federal Policy for the Protection of Human Subjects (1991).

Data Availability. The datasets generated during and/or analyzed during the current study are not publicly available due to a data licensing agreement with the Centers for Medicare \& Medicaid Services (CMS).

Open Access. This article is licensed under a Creative Commons Attribution-NonCommercial 4.0 International License, which permits any non-commercial use, sharing, adaptation, distribution and reproduction in any medium or format, as long as you give appropriate credit to the original author(s) and the source, provide a link to the Creative Commons licence, and indicate if changes were made. The images or other third party material in this article are included in the article's Creative Commons licence, unless indicated otherwise in a credit line to the material. If material is not included in the article's Creative Commons licence and your intended use is not permitted by statutory regulation or exceeds the permitted use, you will need to obtain permission directly from the copyright holder. To view a copy of this licence, visit http://creativecommons.org/licenses/by$\mathrm{nc} / 4.0 /$.

\section{REFERENCES}

1. Centers for Disease Control and Prevention. Influenza (Flu): what you need to know. 2018. https:// www.cdc.gov/flu/keyfacts.htm. Accessed Nov 21, 2018.

2. World Health Organization. Fact sheets: detail: influenza (seasonal). 2018. https://www.who.int/ news-room/fact-sheets/detail/influenza-(seasonal). Accessed Nov 21, 2018.

3. Centers for Disease Control and Prevention. Estimated influenza illnesses, medical visits, and hospitalizations averted by vaccination in the United States. 2018. https://www.cdc.gov/flu/about/ disease/2016-17.htm. Accessed Nov 21, 2018.

4. Putri WCWS, Muscatello DJ, Stockwell MS, Newall AT. Economic burden of seasonal influenza in the United States. Vaccine. 2018;36(27):3960-6.

5. Karve S, Misurski D, Herrera-Taracena G, Davis KL. Annual all-cause healthcare costs among influenza patients with and without influenza-related complications: analysis of a United States managed care database. Appl Health Econ Health Policy. 2013;11(2):119-28.

6. Mauskopf J, Klesse M, Lee S, Herrera-Taracena G. The burden of influenza complications in different high-risk groups: a targeted literature review. J Med Econ. 2013;16(2):264-77.

7. Ghebrehewet S, MacPherson P, Ho A. Influenza. BMJ. 2016;355:i6258.

8. Centers for Disease Control and Prevention. Who is at high risk for flu complications. 2018. https:// www.cdc.gov/flu/about/disease/high_risk.htm. Accessed Nov 21, 2018.

9. European Centre for Disease Prevention and Control. Risk groups for severe influenza. 2018. https:// ecdc.europa.eu/en/seasonal-influenza/preventionand-control/vaccines/risk-groups. Accessed Nov 21, 2018.

10. Mauskopf J, Klesse M, Lee S, Herrera-Taracena G. The burden of influenza complications in different high-risk groups: a targeted literature review. J Med Econ. 2013;16(2):264-77.

11. Uyeki TM, Bernstein HH, Bradley JS, et al. Clinical practice guidelines by the Infectious Diseases Society of America: 2018 update on diagnosis, treatment, chemoprophylaxis, and institutional outbreak management of seasonal influenza. Clin Infect Dis. 2019;68(6):e1-e47.

12. Centers for Disease Control and Prevention (CDC). Prevention and control of seasonal influenza with vaccines. Recommendations of the Advisory Committee on Immunization Practices-United States, 2013-2014. MMWR Recomm Rep. 2013;62(RR-07): $1-43$. 
13. Lu PJ, O'Halloran A, Ding H, Srivastav A, Williams WW. Uptake of influenza vaccination and missed opportunities among adults with high-risk conditions, United States, 2013. Am J Med. 2016;129(6): 636.e1-11.

14. Centers for Disease Control and Prevention. Frequently asked questions about estimated flu burden: what are seasonal-influenza-related deaths? 2018. https://www.cdc.gov/flu/about/disease/us flu-related_deaths.htm. Accessed Nov 21, 2018.

15. Osterholm MT, Kelley NS, Sommer A, Belongia EA. Efficacy and effectiveness of influenza vaccines: a systematic review and meta-analysis. Lancet Infect Dis. 2012;12(1):36-44.

16. Centers for Disease Control and Prevention. Past reasons vaccine effectiveness. https://www.cdc.gov/ flu/vaccines-work/past-seasons-estimates.html. Accessed June 23, 2020.

17. Webster RG, Govorkova EA. Continuing challenges in influenza. Ann N Y Acad Sci. 2018;1323:115-39.

18. Beard KR, Brendish NJ, Clark TW. Treatment of influenza with neuraminidase inhibitors. Curr Opin Infect Dis. 2018;31(6):514-9.

19. Accessdata.fda.gov. Highlights of prescribing information: Tamiflu. 2011. https://www.accessdata.fda. gov/drugsatfda_docs/label/2011/021087s057lbl. pdf. Accessed Apr 17, 2019.

20. Accessdata.fda.gov. Highlights of prescribing information: Relenza. 2010. https://www.accessdata.fda. gov/drugsatfda_docs/label/2010/021036s025lbl. pdf. Accessed Apr 17, 2019.

21. Accessdata.fda.gov. Highlights of prescribing information: Rapivab. 2014. https://www.accessdata.fda. gov/drugsatfda_docs/label/2014/206426lbl.pdf. Accessed Apr 17, 2019.

22. Parra-Rojas C, Nguyen VK, Hernandez-Mejia G, Hernandez-Vargas EA. Neuraminidase inhibitors in influenza treatment and prevention: is it time to call it a day? Viruses. 2018;10(9):454.

23. Venkatesan S, Myles PR, Leonardi-Bee J, et al. Impact of outpatient neuraminidase inhibitor treatment in patients infected with influenza $\mathrm{A}(\mathrm{H} 1 \mathrm{N1}$ )pdm09 at high risk of hospitalization: an individual participant data metaanalysis. Clin Infect Dis. 2017;64(10):1328-34.

24. Muthuri SG, Venkatesan S, Myles PR, et al. Effectiveness of neuraminidase inhibitors in reducing mortality in patients admitted to hospital with influenza A H1N1pdm09 virus infection: a metaanalysis of individual participant data. Lancet Respir Med. 2014;2(5):395-404.
25. Ison MG. Optimizing antiviral therapy for influenza: understanding the evidence. Expert Rev Anti Infect Ther. 2015;13(4):417-25.

26. Principi N, Camilloni B, Alunno A, Polinori I, Argentiero A, Esposito S. Drugs for influenza treatment: is there significant news? Front Med (Lausanne). 2019;6:109.

27. Centers for Disease Control and Prevention. Influenza antiviral medications: summary for clinicians. 2018. https://www.cdc.gov/flu/professionals/ antivirals/summary-clinicians.htm. Accessed Nov $21,2018$.

28. Lansbury L, Rodrigo C, Leonardi-Bee J, NguyenVan-Tam J, Lim WS. Corticosteroids as adjunctive therapy in the treatment of influenza. Cochrane Database Syst Rev. 2019;2(2):CD010406.

29. Xu X, Blanton L, Elal AIA, et al. Update: influenza activity in the United States during the 2018-19 season and composition of the 2019-20 influenza vaccine. MMWR Morb Mortal Wkly Rep. 2019;68(24):544-51.

30. Fingar K, Liang L, Stocks C. HCUP Statistical Brief \#253. Inpatient hospital stays and emergency department visits involving influenza, 2006-2016. https://www.hcup-us.ahrq.gov/reports/statbriefs/ sb253-Influenza-Hospitalizations-ED-Visits-20062016.jsp\#: :text=During\%2520this\%2520flu\% 2520season $\% 2 \mathrm{C} \% 25207 \% 2 \mathrm{C} 500$,inpatient $\%$ 2520 stays\%2520involving\%2520the\%2520flu. Accessed 17 Apr 2019.

31. Kaiser Family Foundation. Distribution of Medicare beneficiaries by gender. 2018. https://www.kff.org/ medicare/state-indicator/medicare-beneficiaries-bygender $/$ currentTimeframe $=0 \&$ sortModel $=\% 7 \mathrm{~B} \%$ 22colId\%22:\%22Location\%22,\%22sort\%22:\% $22 \mathrm{asc} \% 22 \% 7 \mathrm{D}$. Accessed 17 Apr 2019.

32. Thompson WW, Shay DK, Weintraub E, et al. Mortality associated with influenza and respiratory syncytial virus in the United States. JAMA. 2003;289(2):179-86.

33. Molinari NAM, Ortega-Sanchez IR, Messonnier ML, et al. The annual impact of seasonal influenza in the US: measuring disease burden and costs. Vaccine. 2007;25(27):5086-96.

34. Mullooly JP, Bridges CB, Thompson WW, et al. Influenza- and RSV-associated hospitalizations among adults. Vaccine. 2007;25(5):846-55.

35. Choi WS, Baek JH, Seo YB, et al. Severe influenza treatment guideline. Korean $\mathrm{J}$ Intern Med. 2014;29(1):132-47. 
36. Public Health England. PHE guidance on use of antiviral agents for the treatment and prophylaxis of seasonal influenza. 2019. https://assets. publishing.service.gov.uk/government/uploads/ system/uploads/attachment_data/file/773369/PHE_ guidance_antivirals_influenza.pdf. Accessed Apr 17, 2019.

37. Pockett RD, Watkins J, McEwan P, Meier G. Burden of illness in UK subjects with reported respiratory infections vaccinated or unvaccinated against influenza: a retrospective observational study. PLoS One. 2015;10(8):e0134928.

38. Rothberg MB, Haessler SD. Complications of seasonal and pandemic influenza. Crit Care Med. 2010;38(4 suppl):e91-e9797.

39. Rothberg MB, Haessler SD, Brown RB. Complications of viral influenza. Am J Med. 2008;121(4): 258-64.

40. Venkatesan S, Myles PR, Bolton KJ, et al. Neuraminidase inhibitors and hospital length of stay: a meta-analysis of individual participant data to determine treatment effectiveness among patients hospitalized with nonfatal 2009 pandemic influenza A(H1N1) virus infection. J Infect Dis. 2020;22: 356-66.

41. Chen L, Han X, Li Y, Zhang C, Xing X. Impact of early neuraminidase inhibitor treatment on clinical outcomes in patients with influenza B-related pneumonia: a multicenter cohort study. Eur J Clin Microbiol Infect Dis. 2020;39(7):1231-8.

42. National Foundation for Infectious Diseases. Flu in adults age 65 years and older: what are the risks? 2018. https://www.adultvaccination.org/vpd/ influenza/influenza-65-infographic. Accessed Nov 21, 2018.

43. Vestergaard LS, Nielsen J, Krause TG, et al. Excess all-cause and influenza-attributable mortality in Europe, December 2016 to February 2017. Euro Surveill. 2017;22(14):30506.

44. v d Hoeven AM, Scholing M, Wever PC, Fijnheer R, Hermans M, Schneeberger PM. Lack of discriminating signs and symptoms in clinical diagnosis of influenza of patients admitted to the hospital. Infection. 2007;35(2):65-68.

45. Dugas AF, Valsamakis A, Atreya MR, et al. Clinical diagnosis of influenza in the ED. Am J Emerg Med. 2015;33(6):770-5.

46. Dimitrijević D, Ilić D, Rakić Adrović S, et al. Predictors of hospitalization and admission to intensive care units of influenza patients in Serbia through four influenza seasons from 2010/2011 to 2013/2014. Jpn J Infect Dis. 2017;70(3):275-83.

47. Govaert TME, Thijs CTM, Masurel N, Sprenger MJW, Dinant GJ, Knottnerus JA. The efficacy of influenza vaccination in elderly individuals: a randomized double-blind placebo-controlled trial. JAMA. 1994;272(21):1661-5.

48. Centers for Disease Control and Prevention, National Center for Immunization and Respiratory Diseases. The flu: caring for someone sick at home (2010). Policy brief. https://www.cdc.gov/flu/pdf/ freeresources/general/influenza_flu_homecare_ guide.pdf.

49. Dobson J, Whitley RJ, Pocock S, Monto AS. Oseltamivir treatment for influenza in adults: a metaanalysis of randomised controlled trials. Lancet. 2015;385(9979):1729-37.

50. Jefferson T, Jones MA, Doshi P, et al. Neuraminidase inhibitors for preventing and treating influenza in healthy adults and children. Cochrane Database Syst Rev. 2014;4:CD00965.

51. Marty FM, Vidal-Puigserver J, Clark C, et al. Intravenous zanamivir or oral oseltamivir for hospitalised patients with influenza: an international, randomised, double-blind, double-dummy, phase 3 trial. Lancet Respir Med. 2017;5(2):135-46.

52. Butler CC, van der Velden AW, Bongard E, et al. Oseltamivir plus usual care versus usual care for influenza-like illness in primary care: an open-label, pragmatic, randomised controlled trial. Lancet. 2020;395(10217):42-52. 